\title{
Derivatization of Tributyltin with Sodium Tetrakis(4-fluorophenyl)- borate for Sensitivity Improvement of Tandem Mass Spectrometry
}

\author{
Shinji TsunoI, ${ }^{\dagger}$ Hirotaka ShIOJI, and Minoru TanaKa \\ Research Center for Environmental Preservation, Osaka University, Suita, Osaka 565-0871, Japan
}

\begin{abstract}
Derivatization of tributyltin for tandem mass spectrometry is described. Tributyltin (TBT) and triphenyltin (TPT) were derivatized with sodium tetrakis(4-fluorophenyl)borate. After optimization of their MS/MS conditions, derivatization conditions were examined. Under the optimum conditions using in-situ derivatization, the calibration curves for the TBT and TPT were linear in the ranges of $0.4-200$ and 1.2-200 pg of Sn, respectively. The detection limits for TBT and TPT were 0.07 and $0.43 \mathrm{pg}$ of $\mathrm{Sn}$, respectively. In the case of TBT, the detection limit with 4-fluorophenylation was improved about five times compared with that with pentylation $(0.35 \mathrm{pg})$. This improvement is ascribed to the bonddissociation energy of Sn-aryl being stronger than that of Sn-alkyl. Namely, the selective fragmentation of 4 fluorophenyl TBT resulted in high sensitivity. The relative recoveries of TBT and TPT from seawater were 99 and $109 \%$, respectively. The method was successfully applied to the seawater samples.
\end{abstract}

(Received September 16, 2003; Accepted November 10, 2003)

\section{Introduction}

In the field of chromatography, detection-oriented derivatization is an important technique for enhancing the response and selectivity of the analytes to the detector. ${ }^{1}$ For GC analysis of polar analytes, derivatization has played an especially important role. ${ }^{1-3}$ The derivatizations for GC analysis can improve the response and selectivity together with the chromatographic properties of the polar analytes. Various derivatizing reagents have been developed to enhance the detector responses of the polar analytes. Fluorine-containing reagents have been extensively used for derivatization reactions such as acylation, alkylation, silylation and condensation, and the resulting derivatives are selectively and sensitively detected with an electron-capture detector. ${ }^{4}$ They also serve effectively as derivatizing reagents for negative ion chemical ionization-mass spectrometry (MS). ${ }^{5-12}$ For nitrogen-phosphorous detection, some reagents are reported to form nitrogen-containing derivatives. ${ }^{13-16}$ For electron ionization (EI)-MS detection, selective fragmentation of the derivative can provide sensitive detection. It is well-known that the $t$-butyldimethylsilylation of hydroxylic compounds is sensitive to EI-MS detection due to its selective fragmentation of the $t$-butyl group. ${ }^{17,18}$

Organotins can generally be analyzed by GC with selective detection after various derivatizations, such as hydrogenation, ethylation, propylation and pentylation. ${ }^{19-21} \mathrm{We}$ previously reported that the detection limit of organotins after pentylation by GC-ion trap tandem mass spectrometry (MS/MS) is about two orders of magnitude lower than that by GC-MS.22 In the tandem mass chromatogram, the peak intensities of three pentylated phenyltins were several times stronger than those of

$\dagger$ To whom correspondence should be addressed.

E-mail: tsunoi@epc.osaka-u.ac.jp three pentylated butyltins (Fig. 1). We considered that the introduction of phenyl group to TBT enhances the intensity because the derivative produced by the arylation of TBT has a structure similar to that produced by the pentylation of monophenyltin (MPT). Here, we report a 4-fluorophenyl derivatization for the determination of tributyltin (TBT) by GCMS/MS.

\section{Experimental}

\section{Materials}

Tributyltin (TBT) chloride and triphenyltin (TPT) chloride were purchased from Wako (Osaka, Japan) and Strem Chemicals (Bischeim, France), respectively. Sodium tetraphenylborate and sodium tetrakis(4-fluorophenyl)borate

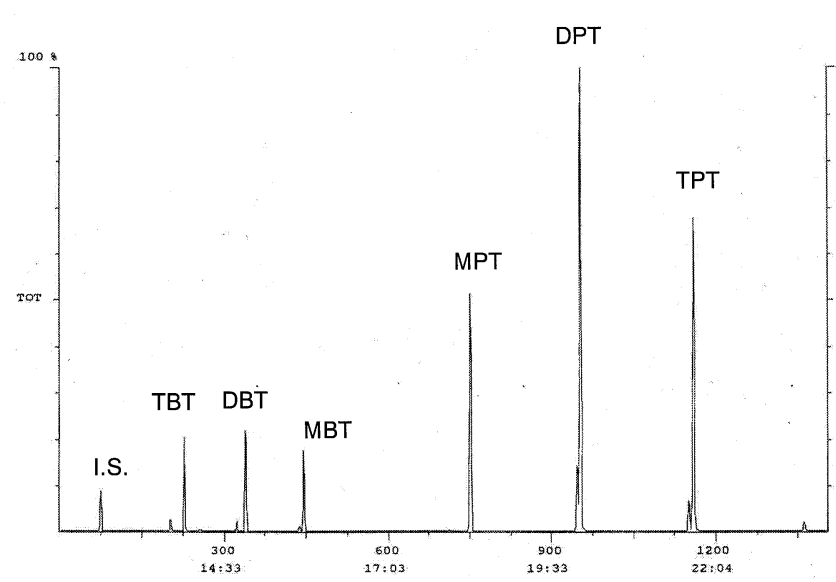

Fig. 1 GC-MS/MS chromatogram of pentylated organotins. 
Table 1 MS/MS conditions of 4-fluorophenylated organotins

\begin{tabular}{cccccc}
\hline Segment & $\begin{array}{c}\text { Retention } \\
\text { time/min }\end{array}$ & Organotins & $\begin{array}{c}\text { Precursor } \\
\text { ion }(m / z)\end{array}$ & $\begin{array}{c}\text { Product } \\
\text { ion }(m / z)\end{array}$ & $\begin{array}{c}\text { CID voltage } / ~ \\
\text { V }\end{array}$ \\
\hline \multirow{2}{*}{1} & 10.62 & TBT-d $d_{27}$ & 347 & 283 & 0.75 \\
& 10.72 & TBT & 329 & 273 & 0.75 \\
2 & 14.38 & Hexyl TPT & 351 & 197 & 1.4 \\
3 & 14.95 & TPT-d 15 & 379 & 202 & 1.2 \\
& 15.03 & TPT & 369 & 197 & 1.2 \\
\hline
\end{tabular}

a. Excitation time: $15 \mathrm{~ms}$ for TBT and hexyl TPT (IS), $24 \mathrm{~ms}$ for TPT.

dihydrate were obtained from Dojindo (Kumamoto, Japan), and other tetraarylborates and 4-fluorophenylmagnesium bromide from Aldrich (Milwaukee, WI, USA). Pesticide grade solvents and other chemicals were from Wako or Kishida Chemical (Osaka, Japan). Water was processed with a Milli-Q VOC system (Millipore, Bedford, MA, USA). Silica gel (BW127ZH, $100-270$ mesh) was a product of Fuji Silysia (Aichi, Japan). Organotin standard solution was prepared according to the previously reported method. ${ }^{22}$

\section{4-Fluorophenyl derivatives of TBT and TPT}

Standard compounds, 4-fluorophenyl TBT and TPT, were synthesized by the Grignard reaction in our laboratory. The products were purified by column chromatography on silica gel and identified by GC-MS and ${ }^{1} \mathrm{H}-$ and ${ }^{13} \mathrm{C}-\mathrm{NMR}$ recorded on a JEOL JNM-GSX-400. Chemical shifts were referenced to the signal of tetramethylsilane.

4-Fluorophenyl TBT: ${ }^{1} \mathrm{H}-\mathrm{NMR}\left(\mathrm{CDCl}_{3}\right) \delta 0.88(\mathrm{t}, J=7.3 \mathrm{~Hz}$, 9H), 1.04 (m, 6H), 1.32 (sextet, $J=7.3 \mathrm{~Hz}, 6 \mathrm{H}), 1.53(\mathrm{~m}, 6 \mathrm{H})$, $7.03(\mathrm{~m}, 2 \mathrm{H}), 7.41(\mathrm{~m}, 2 \mathrm{H}) ;{ }^{13} \mathrm{C}-\mathrm{NMR}\left(\mathrm{CDCl}_{3}\right) \delta 9.66(J=7.3$ and $335.5 \mathrm{~Hz}), 13.66,27.34(J=57.0 \mathrm{~Hz}), 29.04(J=20.2 \mathrm{~Hz})$, $115.10(J=19.3 \mathrm{~Hz}), 136.71(J=3.7 \mathrm{~Hz}), 137.82(J=6.4 \mathrm{~Hz})$, $163.21(J=246.4 \mathrm{~Hz})$.

4-Fluorophenyl TPT: ${ }^{1} \mathrm{H}-\mathrm{NMR}\left(\mathrm{CDCl}_{3}\right) \delta 7.10(\mathrm{~m}, 2 \mathrm{H}), 7.37$ $7.43(\mathrm{~m}, 9 \mathrm{H}), 7.50-7.65(\mathrm{~m}, 8 \mathrm{H}) ;{ }^{13} \mathrm{C}-\mathrm{NMR}\left(\mathrm{CDCl}_{3}\right) \delta 115.90$ $(J=19.3 \mathrm{~Hz}), 128.72(J=26.2 \mathrm{~Hz}), 129.27(J=6.0 \mathrm{~Hz}), 132.95$ $(J=3.7 \mathrm{~Hz}), 137.17(J=18.9 \mathrm{~Hz}), 137.64,138.80(J=6.4$ and $21.6 \mathrm{~Hz}), 163.88(J=248.2 \mathrm{~Hz})$.

\section{GC-MS conditions}

All analyses were performed with a Finnigan MAT GCQ (San Jose, CA, USA) ion trap mass spectrometer equipped with a Finnigan GC. The GC column was a DB-5MS (30 $\mathrm{m} \times 0.25$ $\mathrm{mm}$ i.d., $0.25 \mu \mathrm{m} d_{\mathrm{f}}$, J\&W Scientific, Folsom, CA, USA). The carrier gas was high purity helium $(99.9999 \%)$ with a constant linear velocity of $40 \mathrm{~cm} / \mathrm{s}$. The GC oven temperature was set at $50^{\circ} \mathrm{C}$ for $1 \mathrm{~min}$ and was increased to $290^{\circ} \mathrm{C}$ at $20^{\circ} \mathrm{C} / \mathrm{min}$ and held for $7 \mathrm{~min}$ (total analysis time, $20 \mathrm{~min}$ ). The ion source, injection and transfer-line temperatures were set at 200, 270 and $290^{\circ} \mathrm{C}$, respectively. All injections were carried out in the splitless mode with the split vent closed for $1 \mathrm{~min}$. The mass spectrometer was operated in the electron ionization mode. The mass range was scanned from 50 to $600 \mathrm{u}$ at $0.5 \mathrm{~s} / \mathrm{scan}$ for the full scan mode. For MS/MS experiments, ion peaks having high abundance and $\mathrm{m} / \mathrm{z}$ in each EI spectrum were selected as precursor ions. The CID voltage was optimized to obtain the highest abundance of a product ion. The optimum MS/MS conditions are shown in Table 1 . In segments 1 and 3 , the dual MS/MS mode was used for the isolation and collision of two precursor ions. For quantitation, the product ions were monitored by the selected reaction monitoring (SRM) mode.
Table 2 Effect of derivatizing reagents on GC-MS sensitivity

\begin{tabular}{lcc}
\hline \multirow{2}{*}{ Derivatization } & \multicolumn{2}{c}{ Peak area ratio } \\
\cline { 2 - 3 } & TBT & TPT \\
\hline Phenylation & 4.0 & 1.0 \\
4-Chlorophenylation & 2.4 & 0.56 \\
4-Fluorophenylation & 3.9 & 1.1 \\
p-Tolylation & 3.7 & 0.73 \\
4-Methoxyphenylation & 2.0 & 0.47 \\
\hline
\end{tabular}

The other MS/MS operating parameters were as follows: isolation time, $8 \mathrm{~ms}$; excitation time, $15 \mathrm{~ms}$ for TBT and $24 \mathrm{~ms}$ for TPT.

\section{Calibration curves}

To $50 \mathrm{ml}$ of pure water spiked with the standard solution of the organotins $(5$ or $10 \mu \mathrm{l})$ and the surrogate solution $(10 \mu \mathrm{l})$ in a 100-ml separatory funnel were added toluene $(10 \mathrm{ml})$ and sodium tetrakis(4-fluorophenyl)borate $(60 \mathrm{mg})$. The mixture was then shaken at room temperature for $60 \mathrm{~min}$. After phase separation by adding $\mathrm{NaCl}(5 \mathrm{~g})$ to the mixture, the organic layer was evaporated to $0.4 \mathrm{ml}$. Internal standard solution $(0.1$ $\mathrm{ml}$ ) was added to the concentrate, and then $2 \mu \mathrm{l}$ of this solution was subjected to the GC-MS apparatus. We also examined the quantitative calibration under the derivatization at $40^{\circ} \mathrm{C}$ and confirmed the same performance as that under the derivatization at room temperature. The derivatization at $40^{\circ} \mathrm{C}$ was performed using a temperature-controlled shaker (Bio-Shaker BR-30L, TAITEC, Saitama, Japan).

The organotins were quantified by comparing the peak areas of the organotins with those of the internal standards (hexyl TPT) or the corresponding surrogate compounds. The concentration and detection limits of TBT and TPT given in this paper are expressed as the amount of Sn. Organotin chlorides and their derivatives are indicated by the same abbreviations.

\section{Sample preparation}

Seawater samples were collected from Osaka Bay (Nishinomiya Port, Osaka North Port and Osaka South Port). Except for performing the derivatization at $40^{\circ} \mathrm{C}$, the seawater samples were treated as described for the calibration curves. The absolute recovery was calculated by comparing the peak area of the organotin with that of the internal standard (hexyl TPT). The relative recovery was calculated by calibrating the absolute recovery with the recovery of the corresponding surrogate compound.

\section{Results and Discussion}

\section{Derivatization with tetraarylborates}

Tetraalkylborates such as tetraethylborate ${ }^{23}$ and tetrapropylborate ${ }^{24}$ have been used to derivatize organometallic compounds, whereas the derivatizations with tetraarylborates are restricted to the derivatization of mercury with tetraphenylborate. ${ }^{25,26}$ Little attention has been given to the arylation of organotins. ${ }^{26}$ First of all, we performed the derivatizations of TBT and TPT under in-situ derivatization conditions like those used with tetraethylborate. Five tetraarylborates, i.e., tetraphenylborate, tetrakis(4chlorophenyl)borate, tetrakis(4-fluorophenyl)borate, tetrakis( $p$ tolyl)borate, and tetrakis(4-methoxyphenyl)borate, were 

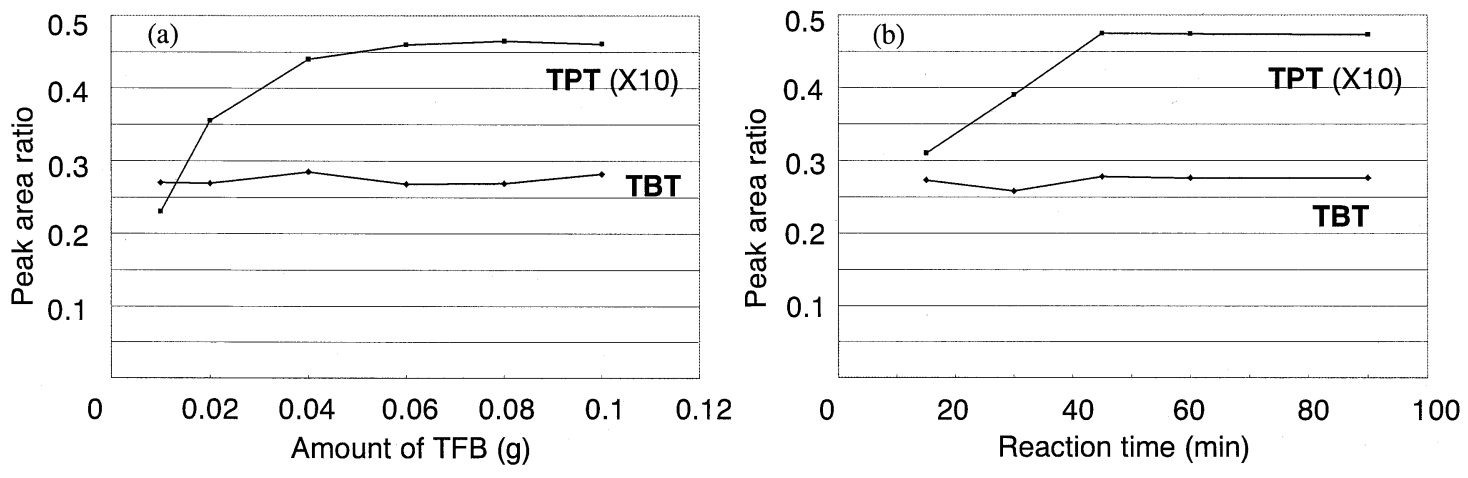

Fig. 2 Optimization of derivatization conditions using pure water $(50 \mathrm{ml})$.

Table 3 Quantitative calibration

\begin{tabular}{ccccc}
\hline & $\begin{array}{c}\text { Calibration } \\
\text { range/pg }\end{array}$ & $\begin{array}{c}\text { Correlation } \\
\text { coefficient, } R\end{array}$ & $\begin{array}{c}\text { Detection } \\
\text { limit } / p g\end{array}$ & RSD $^{\mathrm{b}}, \%$ \\
\hline TBT & $0.4-200$ & 0.9999 & 0.07 & 4.0 \\
TPT & $1.2-200$ & 0.9998 & 0.43 & 2.8 \\
\hline
\end{tabular}

a. Calculated as standard deviation $\times t$, where $t=1.860$ from one-side $t$-distribution at $95 \%$ confidence level $(n=9$, at $0.4 \mathrm{pg}$ for TBT and 2 pg for TPT).

b. Relative standard deviation at $20 \mathrm{pg}(n=5)$.

evaluated as derivatizing reagents. A water sample $(50 \mathrm{ml})$ containing TBT and TPT (100 ng each) was allowed to derivatize in the presence of the derivatizing reagent $(0.01 \mathrm{~g})$ and toluene $(10 \mathrm{ml})$. After shaking for $30 \mathrm{~min}$, the organic phase that separated was concentrated to $0.4 \mathrm{ml}$. Internal standard (hexyl TPT) solution was added to the concentrate and the mixture then subjected to GC-MS. The reactivities of tetraarylborates to TBT and TPT were evaluated by their peak area ratios to the internal standard. The results are shown in Table 2. High peak area ratios were obtained for phenylation and 4-fluorophenylation. In the case of phenylation, however, monophenyltin, diphenyltin, and triphenyltin gave the same derivative, tetraphenyltin. We considered that the derivatizing reagent of choice was tetrakis(4-fluorophenyl)borate (TFB).

\section{Derivatization conditions for 4-fluorophenylation}

After optimization of the MS/MS conditions (Table 1), we investigated the derivatization conditions, the amount of TFB and the reaction time, using above mentioned procedure and MS/MS detection. The amount of TFB was changed over the range of 0.01 to $0.1 \mathrm{~g}$. TBT showed a constant peak area ratio in the range of TFB examined (Fig. 2a). The peak area ratio of TPT increased with an increase in the amount of TFB. This low reactivity of TPT clearly reflects the steric effect of the phenyl group. Further experiments were performed using $0.06 \mathrm{~g}$ of TFB. Under these conditions, the reaction time was examined. The fluorophenylation of TBT was completed within $15 \mathrm{~min}$, but a reaction time of $60 \mathrm{~min}$ was necessary to ensure the complete derivatization of TPT (Fig. 2b). Under the optimum conditions, the derivatization yields of TBT and TPT judged by the synthesized standards were almost quantitative.

\section{Quantitative analysis}

We evaluated the quantitative calibration and detection limits in the range of $0.4-200 \mathrm{pg}$ (Table 3). TBT showed good linearity, having an $R$ value of 0.9999 in the range of $0.4-200$

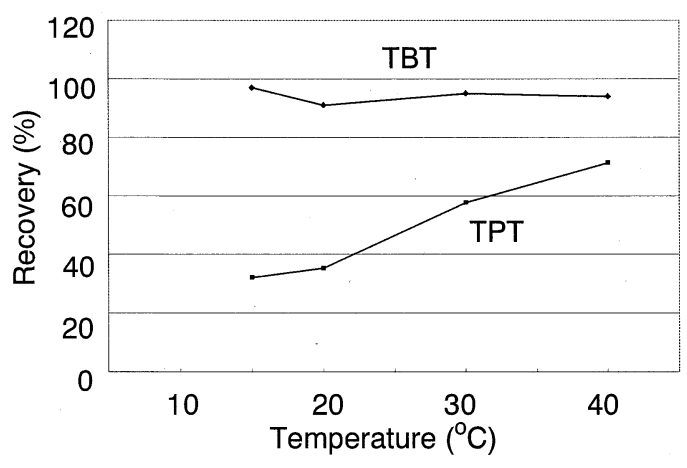

Fig. 3 Optimization of derivatization conditions using seawater.

pg. TPT also showed good linearity, having an $R$ value of 0.9998 in the range of $1.2-200 \mathrm{pg}$. The detection limits obtained from the standard deviations (at $0.4 \mathrm{pg}$ for TBT and 2 $\mathrm{pg}$ for TPT) were $0.07 \mathrm{pg}$ for TBT and $0.43 \mathrm{pg}$ for TPT. According to our expectations, the fluorophenylation improved the detection limit of TBT by several times compared with that of pentyl TBT $(0.35 \mathrm{pg}) .^{22}$ The reproducibilities of this method were found to be $4.0 \%$ for TBT and $2.8 \%$ for TPT at $100 \mathrm{ng} / \mathrm{l}$ for five replicates.

\section{Recovery from seawater sample}

This method was applied to the recoveries of TBT and TPT from seawater spiked at $200 \mathrm{ng} / \mathrm{l}$ level. However, the absolute recovery of TPT resulted in a low level $(38 \%)$. To improve the recovery of TPT, the derivatization conditions were investigated again. Increasing the amount of TFB resulted in no improvement of TPT recovery where large amounts of tris(4fluorophenyl)boroxin and 4,4'-difluorobiphenyl were formed. The effect of the temperature was investigated in the range of $15-40^{\circ} \mathrm{C}$. The reaction temperature has a significant effect on the recovery of TPT (Fig. 3). The recovery of TPT was improved to $69 \%$ at $40^{\circ} \mathrm{C}$. We confirmed that the analytical performance at $40^{\circ} \mathrm{C}$ is the same as that at room temperature.

Under the optimal conditions, we evaluated the recoveries of TBT and TPT from seawater samples at two concentration levels, 20 and $200 \mathrm{ng} / \mathrm{l}$ (Table 4). At both concentration levels, TBT was quantitatively recovered with good reproducibility. On the other hand, the absolute recoveries of TPT were about $70 \%$ regardless of the spiked level. However, the relative recoveries of TPT calibrated by the corresponding surrogate were about $100 \%$, with good reproducibility. 
Mass spectra

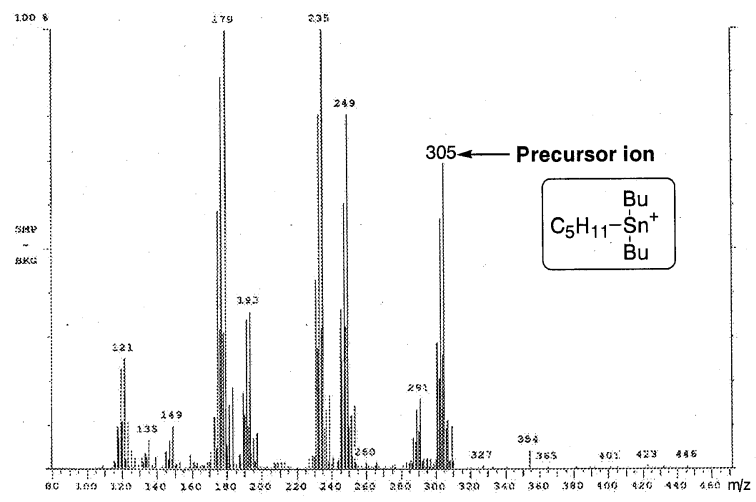

Pentyl TBT

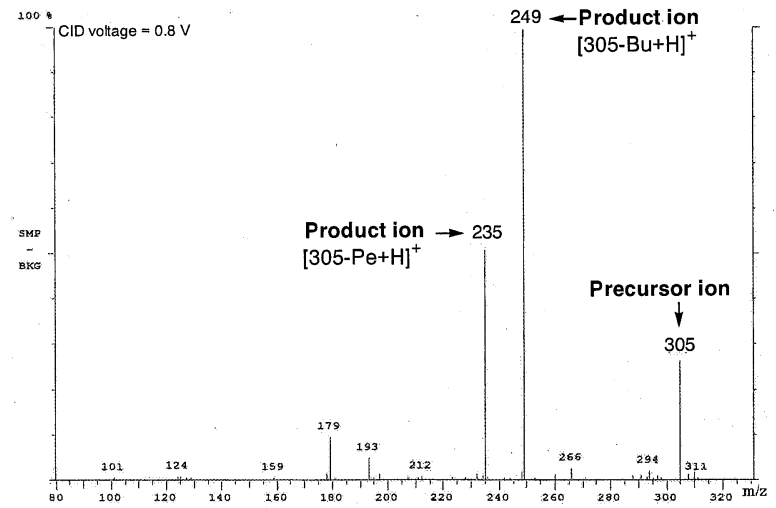

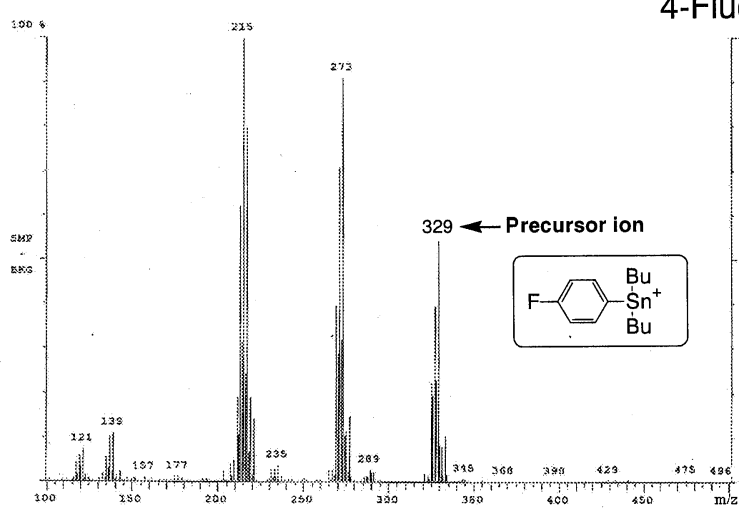

4-Fluorophenyl TBT

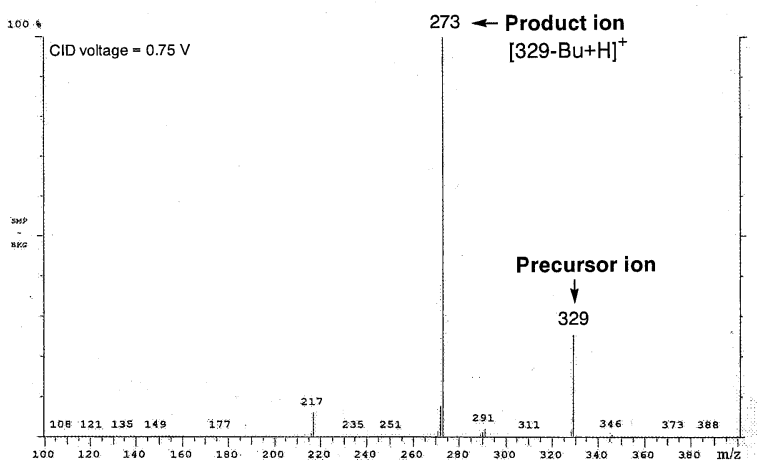

Fig. 4 Mass and tandem mass spectra of pentyl and 4-fluorophenyl TBT.

Table 4 Recoveries of TBT and TPT from seawater ${ }^{\mathrm{a}}$

\begin{tabular}{lcrr}
\hline & \multirow{2}{*}{$\begin{array}{c}\text { Spiked level/ } \\
\text { ng l }^{-1}\end{array}$} & \multicolumn{2}{c}{ Recovery, \% (RSD, \% $)^{\mathrm{b}}$} \\
\cline { 3 - 4 } & 200 & $103(3.8)$ & Absolute \\
\hline \multirow{2}{*}{ TBT } & 20 & $103(6.6)$ & Relative \\
& 200 & $69(7.5)$ & $108(9.8)$ \\
TPT & 20 & $67(3.4)$ & $106(10.0)$ \\
& & & \\
\hline
\end{tabular}

a. Taken from Nishinomiya Port.

b. Relative standard deviation $(n=5)$.

Table 5 Determination of TBT and TPT in seawater ${ }^{a}$

\begin{tabular}{lccc}
\hline & \multicolumn{3}{c}{ Concentration/ng 1-1 $(\mathrm{RSD}, \%)^{\mathrm{b}}$} \\
\cline { 2 - 4 } & Nishinomiya Port & North Port & South Port \\
\hline TBT & $1.1^{\mathrm{c}}(8.5)$ & $1.5^{\mathrm{c}}(5.9)$ & $\begin{array}{c}4.0(5.8) \\
\text { nd }\end{array}$ \\
TPT & nd & nd & nd \\
\hline
\end{tabular}

a. Samples were collected from Osaka Bay.

b. Relative standard deviation $(n=5)$.

c. Estimated value by extrapolating the calibration curve.

Application to seawater sample

Seawater samples were taken from the coast of Osaka Bay. As shown in Table 5, TPT could not be detected in any of the three samples $(\mathrm{DL}=2.5 \mathrm{ng} / \mathrm{l})$. TBT was detected in all seawater samples. The TBT concentrations of the two samples

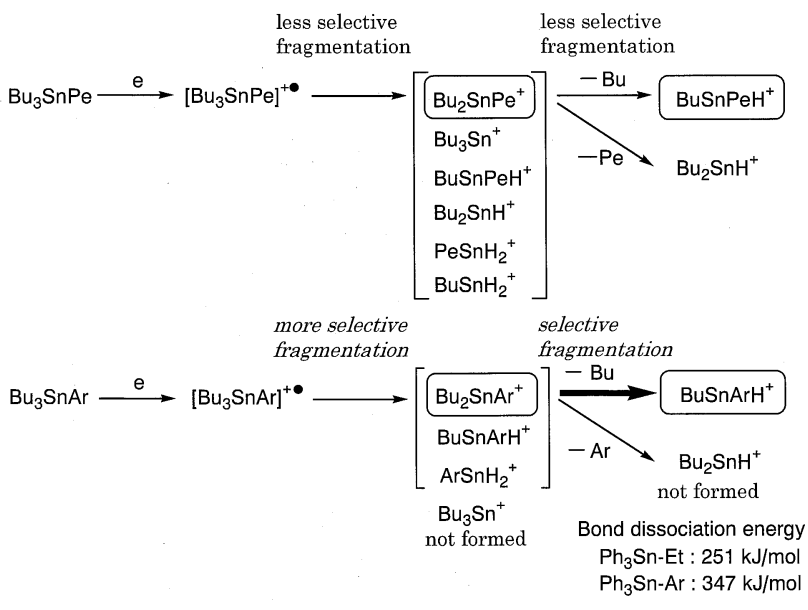

Fig. 5 Selective fragmentation of aryl TBT.

were estimated by extrapolating the calibration curve, because they were outside the calibration range. The reproducibility was between 5.8 and $8.5 \%$ for five replicates. The surrogate compound of TBT was quantitatively recovered, indicating the high reliability of this method.

Sensitivity dependence on structure

Mass and tandem mass spectra of pentylated and 4fluorophenylated TBT are shown in Fig. 4. In the mass spectra, pentyl TBT produced the various fragment ions, whereas 4fluorophenyl TBT produced three fragment ions $\left(\left[\mathrm{FPhBu}_{2} \mathrm{Sn}\right]^{+}\right.$, 
$[\mathrm{FPhBuSnH}]^{+}$and $\left[\mathrm{FPhSnH}_{2}\right]^{+}$. As a result, the precursor ion of 4-fluorophenyl TBT $\left(\left[\mathrm{FPhBu}_{2} \mathrm{Sn}\right]^{+}=329\right)$ has higher intensity than that of the pentyl TBT. In the tandem mass spectra, the precursor ion $\left(\left[\mathrm{PeBu}_{2} \mathrm{Sn}\right]^{+}\right)$of pentyl TBT produced two fragment ions, $\left[\mathrm{Bu}_{2} \mathrm{SnH}\right]^{+}$and $[\mathrm{PeBuSnH}]^{+}$, via the elimination of pentene and butene, respectively. On the other hand, the precursor ion $\left(\left[\mathrm{FPhBu}_{2} \mathrm{Sn}\right]^{+}\right)$of 4-fluorophenyl TBT produced one fragment ion $\left([\mathrm{FPhBuSnH}]^{+}\right)$via the elimination of butene with high selectivity. In other words, the alkyl groups, such as pentyl and butyl groups, dissociate much more easily than the 4-fluorophenyl group. As a result, the fluorophenyl TBT showed a higher intensity than the pentyl compound. This is also true with propylation. These fragmentations can be reasonably explained by the bond dissociation energy (Fig. 5). The bond strength between tin and $\mathrm{sp}^{2}$ carbon is much stronger than that between tin and $\mathrm{sp}^{3}$ carbon. ${ }^{28}$

\section{Conclusions}

We have demonstrated the derivatization of TBT with sodium tetrakis(4-fluorophenyl)borate for MS/MS. With the conventional alkylation method, fragmentation of TBT was uncontrolled and many fragment ions were observed. With 4fluorophenylation, the fragmentation of TBT could be successfully controlled, and a high intensity was observed in the MS/MS mode. Because the derivatizing reagent, TFB, is relatively stable compared to sodium tetraethylborate, its handling is very easy. The recovery of TPT from seawater was not quantitative, due to slow derivatization in seawater. However, the calibration by perdeuterated TPT chloride can provide a precise and accurate measurement. Thus, the MS/MS analysis proved to be effective for its application to environmental water samples.

\section{Acknowledgements}

Thanks are due to the Instrumental Analysis Center, Faculty of Engineering, Osaka University, for assistance in obtaining NMR spectra on a JEOL JNM GSX-400. We thank Dr. H. Harino for the gift of surrogates.

\section{References}

1. K. Blau and J. Halket (ed.), "Handbook of Derivatives for Chromatography", 2nd ed., 1993, Wiley, New York.
2. J. Segura, R. Ventura, and C. Jurado, J. Chromatogr. B, 1998, $713,61$.

3. R. J. Wells, J. Chromatogr. A, 1999, 843, 1.

4. C. F. Poole and A. Zlatkis, Anal. Chem., 1980, 52, 1002A.

5. S. Nakamura, T. H. Sian, and S. Daishima, J. Chromatogr. A, 2001, 919, 275.

6. J. Cheung and R. J. Wells, J. Chromatogr. A, 1997, 771, 203.

7. H. J. Leis, W. Windischhofer, and G. Fauler, J. Chromatogr. B, 2002, 779, 353.

8. D. B. Naritsin, R. L. Boni, and S. P. Markey, Anal. Chem., 1995, 67, 863 .

9. M. L. Selley, J. Chromatogr. B, 1997, 691, 263.

10. J. T. Martin, J. D. Barchas, and K. F. Faull, Anal. Chem., 1982, 54, 1806.

11. J. V. Johnson, R. A. Yost, and K. F. Faull, Anal. Chem., 1984, 56, 1655.

12. T. M. Trainor and P. Vouros, Anal. Chem., 1987, 59, 601.

13. M. J. Bertrand, S. Stefanidis, and B. Sarrasin, J. Chromatogr., 1986, 351, 47.

14. M. Beljean-Leymarie and E. Bruna, Anal. Biochem., 1988, $173,174$.

15. H. S. Shin, S. K. Kim, S. W. Myung, and J. S. Park, Anal. Chem., 1995, 67, 1853.

16. F. E. O. Suliman and Y. Soma, J. Environ. Monit., 2000, 2, 470.

17. T. Ohie, X. W. Fu, M. Iga, M. Kimura, and S. Yamaguchi, J. Chromatogr. B, 2000, 746, 63.

18. M. Kataoka, K. Tsuge, and Y. Seto, J. Chromatogr. A, 2000, 891,295

19. K. M. Attar, Appl. Organomet. Chem., 1996, 10, 317.

20. M. Abalos, J. M. Bayona, R. Companó, M. Granados, C. Leal, and M. D. Prat, J. Chromatogr. A, 1997, 788, 1.

21. M. Takeuchi, K. Mizuishi, and T. Hobo, Anal. Sci., 2000, $16,349$.

22. S. Tsunoi, T. Matoba, H. Shioji, L. T. H. Giang, H. Harino, and M. Tanaka, J. Chromatogr. A, 2002, 962, 197.

23. J. Ashby, S. Clark, and P. J. Craig, J. Anal At. Spectrom., 1988, 3, 735 .

24. T. D. Smaele, L. Moens, R. Dams, P. Sandra, J. V. Eycken, and J. Vandyck, J. Chromatogr. A, 1998, 793, 99.

25. V. Luckow and H. A. Rüssel, J. Chromatogr., 1978, 150, 187.

26. V. Minganti, R. Capelli, and R. De Pellegrini, Fresenius J. Anal. Chem., 1995, 351, 471.

27. M. Azenha and M. T. Vasconcelos, Anal. Chim. Acta, 2002, 458, 231.

28. P. G. Harrison (ed.), "Chemistry of Tin", 1989, Blackie, London. 